\title{
Balancing cyclic $R$-ary Gray codes
}

\author{
Mary Flahive \\ Department of Mathematics \\ Oregon State University, Corvallis, OR 97331, USA \\ flahive@math.oregonstate.edu \\ Bella Bose \\ School of Electrical Engineering and Computer Science \\ Oregon State University, Corvallis, OR 97331, USA \\ bose@eecs.oregonstate.edu
}

Submitted: Sep 26, 2006; Accepted: Apr 17, 2007; Published: Apr 27, 2007

Mathematics Subject Classification: 05A99,05C45,68R10

\begin{abstract}
New cyclic $n$-digit Gray codes are constructed over $\{0,1, \ldots, R-1\}$ for all $R \geq 3$, $n \geq 2$. These codes have the property that the distribution of the digit changes (transition counts) is close to uniform: For each $n \geq 2$, every transition count is within $R-1$ of the average $R^{n} / n$, and for the 2-digit codes every transition count is either $\left\lfloor R^{2} / 2\right\rfloor$ or $\left\lceil R^{2} / 2\right\rceil$.
\end{abstract}

\section{Introduction}

For $R, n \geq 2$, an $\mathbf{n}$-digit $\mathbf{R}$-ary Gray code is a sequence in which each $n$-digit string with digits from the set $\{0,1, \ldots, R-1\}$ occurs exactly once and any two consecutive strings differ in only one digit and the difference equals \pm 1 ; that is, the Lee distance equals 1 , where the Lee distance between two $n$-strings is defined to be

$$
d_{L}\left(a_{1} \ldots a_{n}, b_{1} \ldots b_{n}\right)=\sum_{j=1}^{n} \min \left\{\left|a_{j}-b_{j}\right|, R-\left|a_{j}-b_{j}\right|\right\} .
$$

When the Lee distance between the last and first strings also equals 1, the code is called cyclic. For instance,

$$
10,11,01,00 \text { and } 20,21,22,12,02,01,11,10,00
$$

are cyclic 2-digit $R$-ary Gray codes, with $R=2$ and $R=3$, respectively. 
It is helpful to consider a cyclic Gray code as a Hamiltonian cycle in the $\mathbf{R}$-ary $\mathbf{n}$ cube, the graph whose vertices are all $n$-strings with digits from the set $\{0,1, \ldots, R-1\}$ in which two vertices are adjacent if they differ in only one digit by \pm 1 . Our usual representation of this graph will have the vertices arranged in an $R^{n-1} \times R$ array, where the first $n-1$ digits of a vertex $n$-string are given by its row label and the last digit by its column label. Figure 1 has the Hamiltonian cycles for the cyclic Gray codes in (1).
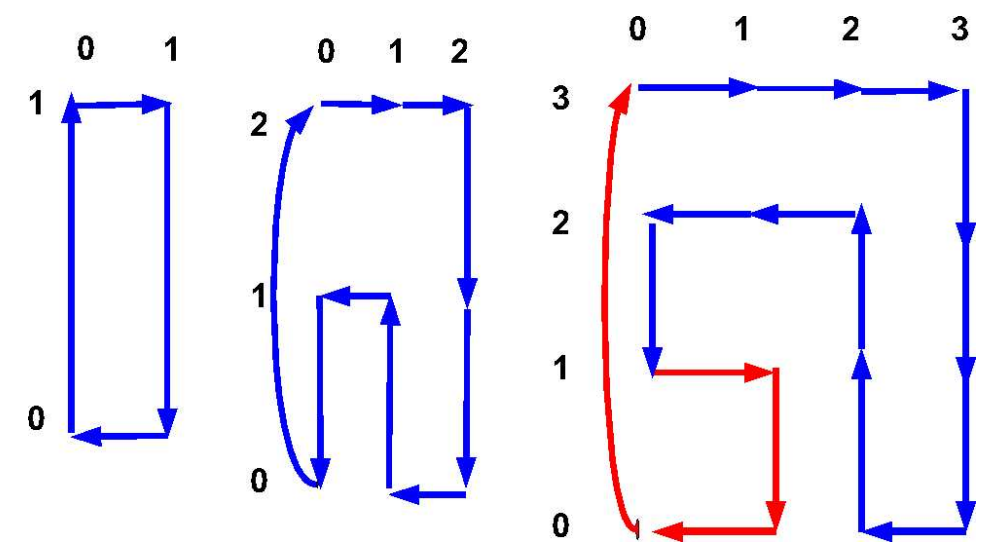

Figure 1: cyclic 2-digit $R$-ary Gray codes for $R=2,3,4$.

The transition sequence of a cyclic Gray code records the successive digit changes in the code, beginning with the change from the first to the second string. For example, the transition sequences for the codes in (1) are:

$$
2,1,2,1 \text { and } 2,2,1,1,2,1,2,1,1 \text {. }
$$

For each $1 \leq j \leq n$, the transition count $T C(j)$ of the digit $j$ is defined to be the number of times $j$ occurs as a transition digit in the code. For each $n, R$ there are many Gray codes, and one favorable characteristic for applications is a relative uniformity in the distribution of transition counts. When all transition counts are equal (this common value must necessarily equal $R^{n} / n$ and $n$ must divide $R^{n}$ ), the code is called totallybalanced or uniform. The binary and quaternary codes in Figure 1 are totally-balanced. In $[7,10,11]$ totally-balanced cyclic $n$-digit binary codes are constructed for every $n$ which is a power of 2 , but there is no published construction of families of totally-balanced codes for arbitrary $R$. One of our results yields totally-balanced quaternary codes. (Refer to Corollary 1.)

When $R^{n}$ is not divisible by $n$, totally-balanced cyclic codes cannot exist and it is reasonable to ask for codes in which every transition count is either $\left\lfloor R^{n} / n\right\rfloor$ or $\left\lceil R^{n} / n\right\rceil$, a phenomenon which we will call well-balanced. When $R$ is even, the cyclicness forces every transition count to be even and so (unlike the cyclic ternary code given above in (1)) for even $R$ every well-balanced cyclic $R$-ary Gray code must be totally-balanced. In [2], the authors asked if a well-balanced (non-cyclic) $n$-digit binary Gray code exists for every $n \geq 2$. Although this continues to be an open question, in [9, Chapter 3] I. 
N. Suparta has obtained further evidence supporting the conjecture. In Section 2 we construct well-balanced 2-digit $R$-ary Gray codes for every $R \geq 2$. The codes depend on the even/odd parity of $R$, and are produced by a natural inductive process in which well-balanced cyclic 2-digit $(R+2)$-ary Gray codes are constructed from the $R$-ary ones.

A cyclic $n$-digit binary Gray code satisfying

$$
|T C(i)-T C(j)| \leq 2 \text { for every pair } i, j,
$$

is referred to as balanced. In [2], G. S. Bhat and C. Savage constructed balanced cyclic $n$-digit (binary) Gray codes for every $n \geq 2$ when they completed some details which remained from the construction of J. P. Robinson and M. Cohn [7]. In a comment added to [2], F. Ruskey noted that T. Bakos [1, pp. 28-37] had much earlier constructed balanced cyclic binary codes; more recently I. N. Suparta [9, Section 3.2] has developed an elegant variant of these constructions. In each, $n$-digit cyclic binary codes are constructed inductively, using four $(n-2)$-digit binary codes.

Non-binary codes have been considered, for example, in $[3,4,5,6,8,9]$, but the question of constructing fairly uniform cyclic $R$-ary Gray codes for a general radix $R>2$ has received far less attention than the binary case. In Section 3 we give a simple inductive construction which we later use in Section 4 to obtain nearly-balanced codes; that is, codes whose transition counts satisfy

$$
\left|T C(j)-\frac{R^{n}}{n}\right|<\rho \quad \text { for all } 1 \leq j \leq n,
$$

where $\rho$ equals $R-1$ or $R-2$, whichever is even. The earlier constructions of well-balanced binary codes relied on delicate combinatorial arguments, whereas our use of $R^{n} / n$ as a reference point has the advantage of allowing simpler proofs which involve only balancing inequalities.

\section{Well-balanced cyclic 2-digit R-ary Codes}

Theorem 1. Let $R \geq 2$. If there exists a cyclic 2-digit $R$-ary Gray code with transition counts $\left\lfloor R^{2} / 2\right\rfloor+a$ and $\left\lceil R^{2} / 2\right\rceil+b$ for some constants $a, b$, then there exists a cyclic 2 -digit $(R+2)$-ary Gray code with transition counts $\left\lfloor(R+2)^{2} / 2\right\rfloor+a$ and $\left.\left\lceil(R+2)^{2} / 2\right\rceil\right\}+b$.

Proof. The given cyclic $R$-ary code can be normalized so that it begins with R-1 0 and ends with the string 00 . (This can be done because at some point there must be consecutive terms of the form $c d ; c \pm 1 d$. The required form is then obtained by translating and reflecting if necessary so that the last term is 00 and the first term is R-1 0 . Since the distribution of transition counts remains the same under these operations, we may assume the original cyclic code has this form.) Therefore, the code corresponds to a Hamiltonian path from R-1 0 to 00 in the $R$-ary square to which the edge from 00 to R-1 0 can be added to form a Hamiltonian cycle.

Labeling the rows of the $(R+2)$-ary square as $R+1 R \ldots 10$ and the columns as 01 $\ldots R+1$, we first construct a path from the vertex $R+10$ to $R-10$ by beginning with the 
vertex $R+10$, proceeding horizontally through all vertices of the form $R+1 \mathrm{i}$, from $\mathrm{R}+1$ 0 to $R+1 R+1$, and then vertically down to $0 R+1$. From there we travel to $0 \mathrm{R}$ and continue by proceeding vertically upward to $\mathrm{R} R$, traveling horizontally over to $\mathrm{R} 0$ and then ending with the edge to R-1 0 . This initial path covers all vertices in which at least one component is either $\mathrm{R}$ or $\mathrm{R}+1$. Its transition sequence is: $R+1$ twos, followed by $R+1$ ones, one 2 , and then $R$ ones, $R$ twos and one 1 , for a total of $2 R+2$ copies of each of 1 and 2. Attaching the given Hamiltonian path from R-1 0 to 00 to the end of this initial segment results in a Hamiltonian path in the $(R+2)$-ary square, and the addition of the edge from 00 to $\mathrm{R}+10$ results in a Hamiltonian cycle. (The quaternary code in Figure 1 was obtained in this way from the binary code, and the diagram in Figure 2 illustrates the general construction.)

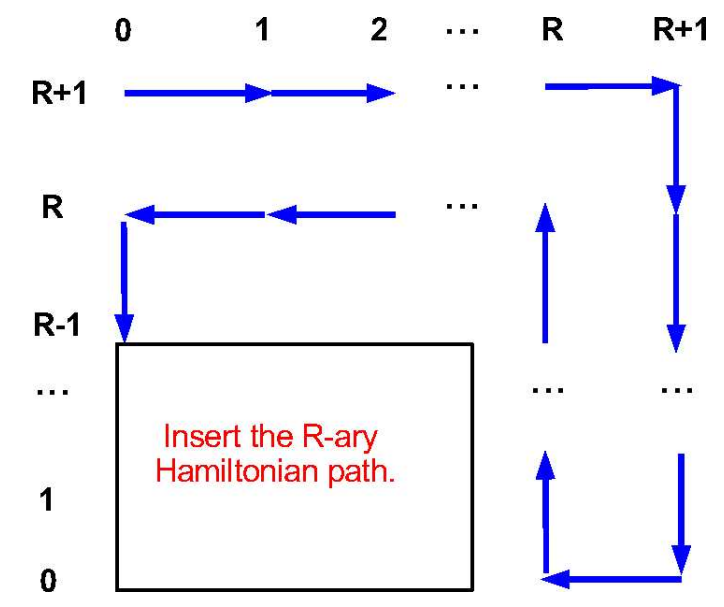

Figure 2: Construction of the initial segment.

Since

$$
\left\lfloor R^{2} / 2\right\rfloor+2 R+2=\left\lfloor(R+2)^{2} / 2\right\rfloor \quad \text { and } \quad\left\lceil R^{2} / 2\right\rceil+2 R+2=\left\lceil(R+2)^{2} / 2\right\rceil,
$$

the code produced is a cyclic 2-digit $(R+2)$-ary Gray code with the required transition counts.

Theorem 2. For any $R \geq 2$ there exists a cyclic 2-digit $R$-ary Gray code which is wellbalanced, and so for even $R$ the code is totally-balanced.

Proof. Consider the statement: For every $n \geq 1$ there exist well-balanced cyclic 2-digit $R$-ary Gray codes for $R=2 n$ and $R=2 n+1$. The codes given in (1) satisfy this statement for $n=1$. Application of Theorem 1 with $a=b=0$ therefore yields the result by induction on $n$. 


\section{Constructing $(n+1)$-digit codes from $n$-digit codes}

One simple way to construct a Hamiltonian path on an $L \times R$ rectangular grid is to traverse successive vertices across rows; that is,

$$
10,11, \ldots, 1 R-1 ; 2 R-1, \ldots, 21,20 \text { etc. }
$$

as illustrated in Figure 3. Notice that the terminal vertex is either $\mathrm{L} 1$ or $\mathrm{L} \mathrm{R}$, according

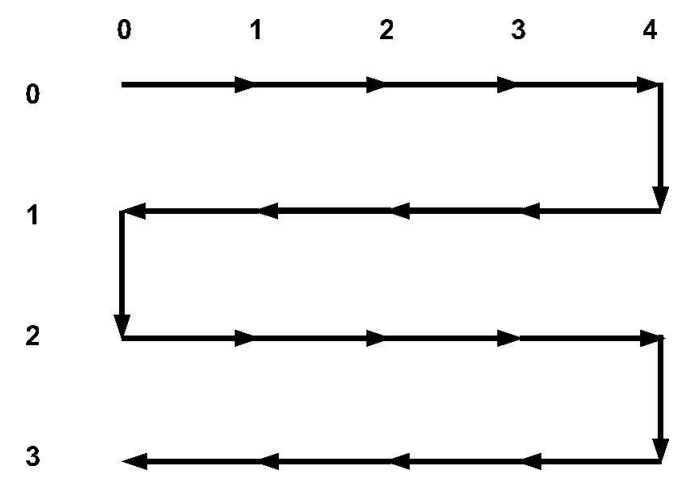

Figure 3: Our basic Hamiltonian path on the $4 \times 5$ grid.

to whether $\mathrm{L}$ is even or odd.

Our construction of nearly-balanced cyclic codes is based on a modification of this simple idea. The construction involves partitioning the row indices of an $R$-ary $(n+1)$ cube into blocks. Specifically, the rows are first indexed by a fixed cyclic $n$-digit $R$-ary Gray code, $a_{1}, a_{2}, \ldots, a_{R^{n}}$, and then are partitioned into $L$ nonempty blocks of consecutive elements, say

$$
B_{1}:=a_{1}, \ldots, a_{i_{1}}, B_{2}:=a_{i_{1}+1}, \ldots, a_{i_{2}}, \ldots, B_{L}:=a_{i_{L-1}+1}, \ldots, a_{R^{n}} .
$$

This idea of using partitions is at least implicit in other published constructions, among them $[2,6,7,9,10]$.

The digit changes from $a_{i_{k}}$ to $a_{i_{k+1}}$ will be called the connecting digits of the partition, and the number of times any digit occurs as a connecting digit will be referred to as its connecting multiplicity. For notational convenience, we assume the code is in a standard form in which the coordinates of the $n$-strings have been permuted if necessary so that 1 is the $L$-th connecting digit, the transition digit from $a_{R^{n}}$ to $a_{1}$.

We use this partition to construct a Hamiltonian path in the $R$-ary $(n+1)$-cube in the following way: First of all, the Hamiltonian path will "respect" the partition $B_{1} \cup \ldots \cup B_{L}$; that is, for every column index $k$ and every block $B_{j}$, the path must traverse the vertices $a_{i_{j}+1} k, a_{i_{j}+2} k, \ldots, a_{i_{j+1}} k$ consecutively either in that direction or in the reverse direction. This allows us to picture the $(n+1)$-cube as an $L \times R$ array in which the rows are indexed by the blocks $B_{1}, \ldots, B_{L}$. 


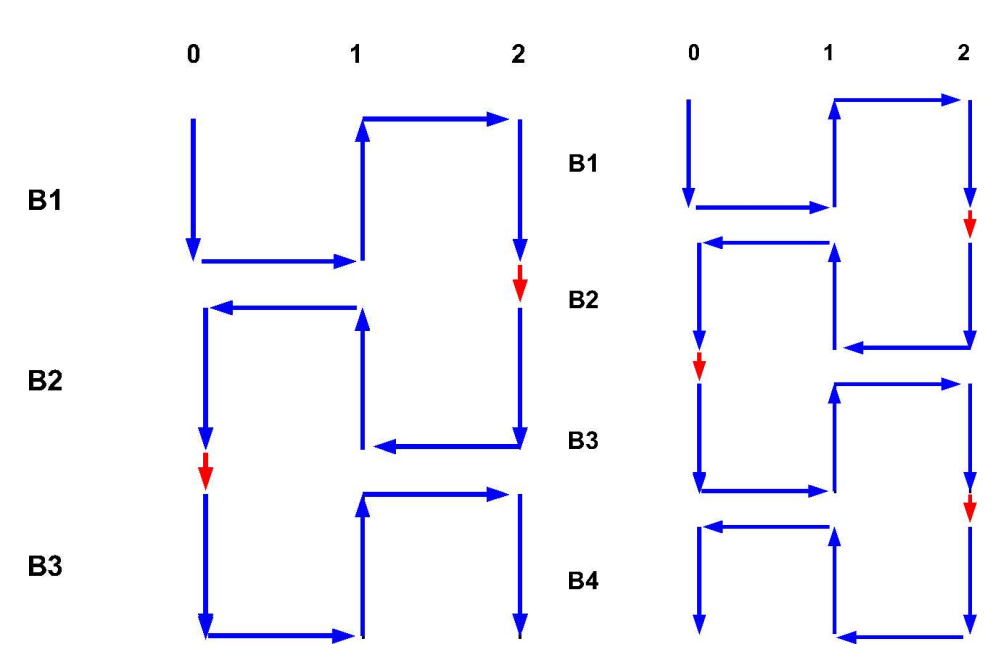

Figure 4: Ternary Hamiltonian paths for three and four blocks.

If $R$ is odd, the construction given in (3) yields a Hamiltonian path on the $R$-ary $(n+1)$-cube (refer to Figure 4$)$, and when the number $L$ of partition-blocks is even number, the addition of the edge from $a_{R^{n}} 0$ to $a_{1} 0$ results in a Hamiltonian cycle.

When $R$ is even, the path can be adjusted to get a Hamiltonian cycle on the $R$-ary $(n+1)$-cube in the following way: Within the given $R$-ary $(n+1)$-cube (with its rows labeled by the cyclic Gray code which has been partitioned by $B_{1} \cup \ldots B_{L}$ and its columns labeled $01 \ldots \mathrm{R}-1)$, consider the $R^{n} \times(R-1)$ grid consisting of the vertices whose last digit is nonzero. Since $R-1$ is odd, the Hamiltonian path given in (3) can be constructed on the blocks of this grid. Its initial vertex is $a_{1} 1$ and the terminal vertex is either $a_{R^{n}}$ R-1 or $a_{R^{n}} 1$, depending on whether $L$ is odd or even. In either event, the terminal vertex is adjacent to $a_{R^{n}} 0$. A Hamiltonian cycle on the $R$-ary $(n+1)$-cube is obtained by appending this edge to the initial path, following through all vertices with zero second digit in the order:

$$
a_{R^{n}} 0, a_{R^{n}-1} 0, \ldots, a_{1} 0,
$$

and then ending with the edge to $a_{1} 1$. This is pictured for $R=4$ in Figure 5 .

Regardless of whether $R$ is even or odd, the construction gives a cyclic $(n+1)$-digit $R$-ary Gray code (provided the number of partition-blocks is even when $R$ is odd). In what follows this will be referred to as the code induced by the partition $B_{1} \cup \ldots B_{L}$.

By arranging the vertices of the (binary) $n$-cube in a grid whose rows are indexed by an $(n-2)$-cube and its columns by 00101101 , most known $n$-digit binary codes can be seen to have this kind of partitioning behavior. When viewed from this perspective of partitions, the combinatorial argument for the binary constructions in $[2,7]$ required some restrictions on the usable partitions; for instance, at least two blocks can have only one element.

The following result gives the distribution of transition counts in codes induced by a partition. 


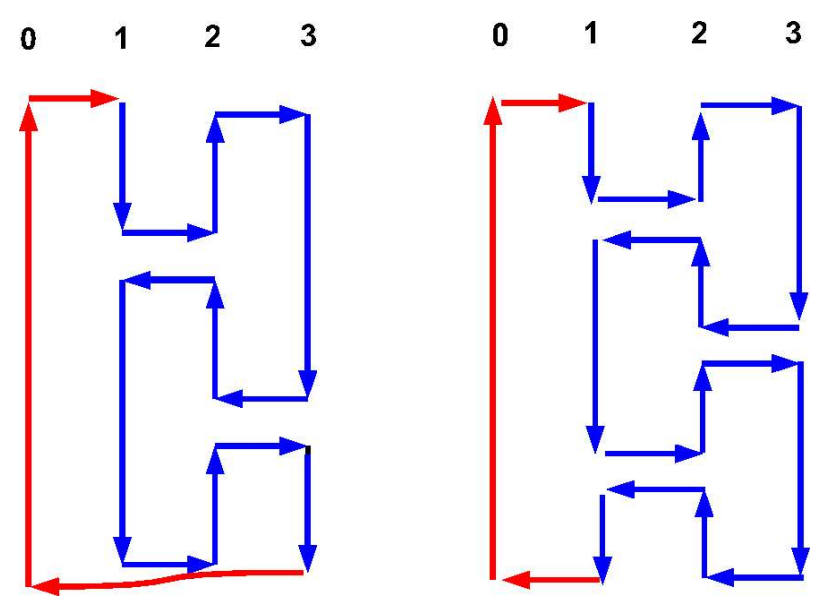

Figure 5: Construction of cyclic 4-ary codes.

Theorem 3. Let $R \geq 3$ be an integer, and set $\rho$ equal to either $R-2$ or $R-1$, whichever is even. Let $a_{1}, a_{2}, \ldots, a_{R^{n}}$ be any cyclic $n$-digit $R$-ary Gray code with transition counts $T_{1}, \ldots, T_{n}$ (where two digit positions have been transposed if necessary so that the digit change from $a_{R^{n}}$ to $a_{1}$ is in the first digit). Let $B_{1} \cup \ldots B_{L}$ be any partition of the code, and $k_{j}$ be the connecting multiplicity of the digit $j$. When $R$ is odd and $L$ is even, the $j$-th transition count of the induced cyclic $(n+1)$-digit Gray code is

$$
T C(j)=\left\{\begin{array}{ll}
R T_{j}-k_{j} \rho & \text { if } j \leq n \\
L \rho & \text { if } j=n+1
\end{array} .\right.
$$

When $R$ is even the transition counts of the induced cyclic $(n+1)$-digit Gray code are

$$
T C(j)=\left\{\begin{array}{ll}
R T_{j}-k_{j} \rho-2 & \text { if } j=1 \\
R T_{j}-k_{j} \rho & \text { if } 1<j \leq n \\
L \rho+2 & \text { if } j=n+1
\end{array} .\right.
$$

Proof. First we consider the contribution from the initial Hamiltonian path. Setting $N:=\rho+1$ (which is odd), the Hamiltonian path on the $R^{n} \times N$ grid has (N-1)L horizontal lines, and so this initial part of the process of forming the Hamiltonian cycle accumulates (N-1)L changes in the digit $n+1$. Every edge within the partition-blocks is traversed in every column, the edge corresponding to the connecting digit from $a_{R^{n}}$ to $a_{1}$ never occurs, and the edge corresponding to every other connecting digit occurs exactly once. Therefore, for every $1<j \leq n$ the digit $j$ changes

$$
N\left(T_{j}-k_{j}\right)+k_{j}=N T_{j}-(N-1) k_{j}
$$

times in this initial segment, and there is one fewer change for $\mathrm{j}=1$.

When $R$ is odd, the cycle is completed by adding one edge which corresponds to a change in the first digit. Since $N=R$ holds in this case, (4) is obtained. 
For even $R$, two horizontal edges are added to complete the cycle, giving

$$
T C(n+1)=(N-1) L+2=L \rho+2 .
$$

As for additional vertical edges, every edge in the original code except for the one corresponding to transitioning from $a_{R^{n}}$ to $a_{1}$ is added once. Since the transition from $a_{R^{n}}$ to $a_{1}$ is a change in the first digit, this gives

$$
T C(1)=(R-1) T_{1}-(R-2) k_{1}-1+T_{1}-1=R T_{1}-k_{1} \rho-2,
$$

and for all $2 \leq j \leq n$,

$$
T C(j)=(R-1) T_{j}-(R-2) k_{j}+T_{j}=R T_{j}-k_{j} \rho .
$$

This proves (5).

We end this section with the construction of a cyclic 3-digit ternary code induced from a partition of the 2-digit ternary code in (1). Since $R$ is odd, the number of partitionblocks must be even. Also, the digit 1 occurs more frequently as a transition digit in the given 2-digit code than 2 (refer to (2)) and so a preferred partition would have 1 occurring at least as often as a connecting digit as 2 does. Consider any 4-block partition in which 2 occurs only once as a connecting digit. (For instance,

$$
B_{1}:=\{20,21\}, B_{2}:=\{22\}, B_{3}:=\{12,02,01\}, B_{4}:=\{11,10,00\}
$$

is such a partition, since the connecting digits are 2,1,1,1 .) From (4), the induced code has

$$
T C(1)=9 ; T C(2)=10 ; T C(3)=8 .
$$

It is not totally balanced but is nearly-balanced since $\left|T C(j)-R^{n} / n\right| \leq 1$ holds for all $j$.

\section{Nearly-balanced cyclic R-ary Gray Codes}

Lemma 1. Let $R \geq 3$ be an integer. If $T$ is any transition count of either a well-balanced 2 -digit $R$-ary code or a nearly-balanced $n$-digit $R$-ary code for $n \geq 3$, then there exists a unique integer $k$ such that $0<k<T$ and

$$
0 \leq R T-k \rho-\frac{R^{n+1}}{n+1}<\rho .
$$

Proof. For $k:=\left\lfloor\left(R T-\frac{R^{n+1}}{n+1}\right) / \rho\right\rfloor$, we see that (6) is satisfied. Rearranging (6),

$$
R T-\frac{R^{n+1}}{n+1}-\rho<k \rho \leq R T-\frac{R^{n+1}}{n+1},
$$

and so $0<k<T$ can be proved by showing

$$
R T-\frac{R^{n+1}}{n+1}-\rho>0 \quad \text { and } \quad R T-\frac{R^{n+1}}{n+1}<T \rho ;
$$


that is,

$$
R T-\frac{R^{n+1}}{n+1}-\rho>0 \text { and } T(\rho-R)+\frac{R^{n+1}}{n+1}>0 .
$$

Considering the case of a well-balanced 2-digit code, every transition count $T$ satisfies

$$
\frac{R^{2}-1}{2} \leq T \leq \frac{R^{2}+1}{2}
$$

giving

$$
R T-\frac{R^{3}}{3}-\rho \geq \frac{R^{3}-9 R+6}{6}>0
$$

for $R \geq 3$, and since $R-\rho$ equals 2 or 1 ,

$$
T(\rho-R)+\frac{R^{3}}{3} \geq \frac{R^{2}+1}{2}(-2)+\frac{R^{3}}{3}=\frac{R^{2}(R-3)-3}{3}
$$

which is positive for all $R \geq 4$. Also, for $R=3, \quad T(\rho-R)+R^{3} / 3=-T+9 \geq 4$ proving (7) for well-balanced 2-digit codes.

It remains to consider the transition counts of a nearly-balanced $n$-digit code with $n \geq 3$; that is,

$$
\left|T-\frac{R^{n}}{n}\right|<\rho,
$$

where we recall that $\rho$ equals either $R-1$ or $R-2$, whichever is even. The first inequality in (7) can be easily checked for $R=n=3$ since $\rho=2$ and the transition counts satisfy $8 \leq T \leq 10$. Defining $a_{n}:=R^{n+1} / n(n+1)$, we observe that for $R, n \geq 3$

$$
\frac{a_{n+1}}{a_{n}}=R\left(1-\frac{2}{n+2}\right)>1,
$$

and so $a_{n} \geq a_{3}=R^{4} / 12$ for all $n \geq 3$. Since $T$ is a transition count of a nearly-balanced $n$-digit code, $\left|T-R^{n} / n\right|<\rho ; T>\frac{R^{n}}{n}-\rho$ and $\rho \leq R-1$ imply

$$
\begin{aligned}
R T-\frac{R^{n+1}}{n+1}-\rho & >R\left(\frac{R^{n}}{n}-\rho\right)-\frac{R^{n+1}}{n+1}-\rho=a_{n}-(R+1) \rho \\
& \geq a_{3}-(R+1)(R-1)=\frac{R^{2}\left(R^{2}-12\right)+12}{12}
\end{aligned}
$$

which is positive for $R \geq 4$. The first inequality has already been proved for $R=n=3$. For $R=3$ and $n \geq 4$, we observe that

$$
a_{n}-(R+1) \rho \geq a_{4}-4 \cdot 2=\frac{3^{5}}{20}-8>0 .
$$

This proves the first inequality in (7) for all $R, n \geq 3$. 
For the second inequality: $T<\rho+\frac{R^{n}}{n}$ and $\rho<R$ give

$$
\begin{aligned}
T(\rho-R)+\frac{R^{n+1}}{n+1} & >\left(\rho+\frac{R^{n}}{n}\right)(\rho-R)+\frac{R^{n+1}}{n+1} \\
& =\rho^{2}+\left(\frac{R^{n}}{n}-R\right) \rho-\frac{R^{n+1}}{n(n+1)},
\end{aligned}
$$

a strictly increasing function of $\rho \geq R-2$. Evaluation at $\rho=R-2$ gives

$$
(R-2)^{2}-R(R-2)+R^{n}\left(\frac{R}{n+1}-\frac{2}{n}\right)=-2(R-2)+b_{n}
$$

where $b_{n}:=R^{n}\left(\frac{R}{n+1}-\frac{2}{n}\right)$. We note that

$$
\frac{b_{n+1}}{b_{n}}=R \frac{n}{n+2} \cdot \frac{(n+1) R-2(n+2)}{n R-2(n+1)}
$$

where the restrictions $R \geq 3$ and $n \geq 3$ imply each of the two identified factors is greater than 1 . Therefore, $b_{n} \geq b_{3}$ and so

$$
-2(R-2)+b_{n} \geq-2(R-2)+b_{3}=\frac{3 R^{4}-8 R^{3}-24 R+48}{12},
$$

which is positive for all $R \geq 3$. This proves (7) and so the lemma.

Theorem 4. For every integer $R \geq 3$ and every $n \geq 2$ there exists a nearly-balanced cyclic $n$-digit $R$-ary Gray code.

Proof. Let $R \geq 3$ be a fixed integer. Since every well-balanced code is nearly-balanced, Theorem 2 serves as the base case for a proof by induction on $n \geq 2$.

Let $T_{1}, \ldots, T_{n}$ be the transition counts of any 2-digit well-balanced code or any nearlybalanced $n$-digit code for $n \geq 3$. We will prove there is a partition of this code for which the induced cyclic $(n+1)$-digit code is nearly-balanced.

From Lemma 1 it follows that for each $j=1, \ldots, n$ there exists a unique integer $k_{j}$ with $1 \leq k_{j}<T_{j}$ such that

$$
S_{j}:=R T_{j}-k_{j} \rho-\frac{R^{n+1}}{n+1}
$$

satisfies

$$
0 \leq S_{j}<\rho .
$$

Permute the coordinates of the $n$-digit code if necessary to obtain

$$
\rho>S_{1} \geq S_{2} \geq \ldots \geq S_{n} \geq 0
$$

Since each $1 \leq k_{j}<T_{j}$, the digit $j$ occurs more than $k_{j}$ times as a transition digit in the code and so it can be partitioned into $L:=\sum_{j=1}^{n} k_{j}$ blocks in such a way that each digit $j$ occurs exactly $k_{j}$ times as a connecting digit. Using (8) and $\sum_{j=1}^{n} T_{j}=R^{n}$,

$$
\sum_{j=1}^{n} S_{j}=\frac{R^{n+1}}{n+1}-\rho L .
$$

THE ELECTRONIC JOURNAL OF COMBINATORICS 14 (2007), \#R31 
Let $M:=\left\lfloor\sum_{j=1}^{n} S_{j} / \rho\right\rfloor$. Then (9) implies

$$
0 \leq M<n
$$

Also, by definition of $M, \quad 0 \leq \sum_{j=1}^{n} S_{j}-M \rho<\rho$ holds and so (11) gives

$$
0 \leq \frac{R^{n+1}}{n+1}-\rho(L+M)<\rho
$$

We also note that if

$$
S_{M+1}=\ldots=S_{n}=0
$$

then from (11) and (9),

$$
\frac{R^{n+1}}{n+1}-\rho(L+M)=\sum_{j=1}^{n} S_{j}-M \rho=\sum_{j=1}^{M} S_{j}-M \rho<M \rho-M \rho=0,
$$

contrary to (13). From (9) we therefore have

$$
0<S_{j}<\rho \text { for all } j \leq M+1 \quad \text { and } \quad 0 \leq S_{j}<\rho \text { for all } j>M+1 .
$$

We distinguish two cases, according to whether $R$ is odd or even.

CASE 1: $R \geq 3$ is odd. We will prove there exists a refinement of the above partition for which the induced $(n+1)$-digit code is cyclic and nearly-balanced. Since $R$ is odd, the number $L$ of partition-blocks must be even for the code induced by the partition to be cyclic. Our refinement will have $L^{\prime}$ partition-blocks where $L^{\prime}$ equals either $L+M$ or $L+M+1$, whichever is even, and so $D:=L^{\prime}-L$ connecting digits will be added. Since $D$ equals $M$ or $M+1$ and $1 \leq k_{j}<T_{j}$, by (12) $0 \leq D \leq n$ and each of the first $D$ digits can be chosen one more time as a connecting digit, giving $L^{\prime}$ partition-blocks with multiplicities $k_{1}^{\prime}, \ldots, k_{n}^{\prime}$ satisfying

$$
k_{j}^{\prime}=k_{j}+1 \text { if } j \leq D, \quad k_{j}^{\prime}=k_{j} \text { if } j>D .
$$

According to (4), the first $n$ transition counts of the code induced by this new partition have

$$
T C(j)-\frac{R^{n+1}}{n+1}=\left\{\begin{array}{ll}
S_{j}-\rho & \text { if } j \leq D \\
S_{j} & \text { if } D<j \leq n
\end{array},\right.
$$

which by (14) satisfy the nearly-balanced condition. Also, the last transition count is $T C(n+1)=L^{\prime} \rho$, and (13) implies

$$
\frac{R^{n+1}}{n+1}-\rho<(L+M) \rho \leq T C(n+1) \leq(L+M+1) \rho \leq \frac{R^{n+1}}{n+1}+\rho .
$$

Therefore, the code is nearly-balanced unless

$$
(L+M) \rho=R^{n+1} /(n+1),
$$


which cannot happen since $\rho$ is even and $R$ is odd. This completes the proof for odd $R$.

CASE 2: $R \geq 4$ is even. We refine the partition by adding one more connecting digit for each $j=2,3, \ldots, M+1$. This induces a code with $L+M$ partition-blocks whose first $n$ transition digits satisfy

$$
T C(j)-\frac{R^{n+1}}{n+1}=\left\{\begin{array}{ll}
S_{1}-2 & \text { if } j=1 \\
S_{j}-\rho & \text { if } 1<j \leq M+1 \\
S_{j} & \text { if } M+1<j \leq n
\end{array} .\right.
$$

As in Case 1, (14) implies the nearly-balanced condition for all $T C(j)$ with $j \leq n$. Also, since $T C(n+1)=(L+M) \rho+2$, from (13) we have

$$
-\rho \leq-2 \leq \frac{R^{n+1}}{n+1}-T C(n+1)<\rho-2<\rho,
$$

and so the code is nearly-balanced unless $\rho=2$ (and so $R=4$ ) and

$$
T C(n+1)=\frac{R^{n+1}}{n+1}+2 .
$$

For this special case, consider any modification of the last partition which has one less occurrence of 1 as a connecting digit. This changes only the first and last transition counts to

$$
T C(1)+2=S_{1}+\frac{R^{n+1}}{n+1} \quad \text { and } \quad T C(n+1)-\rho=T C(n+1)-2=\frac{R^{n+1}}{n+1},
$$

respectively, resulting in a nearly-balanced code.

We next apply this theorem to the case when $n$ divides $R^{n}$.

Corollary 1. Let $R \geq 3$ and $n \geq 2$ be integers. If $n$ divides $R^{n}$, then there exists a cyclic $n$-digit $R$-ary Gray code whose transition counts $T C(1), \ldots, T C(n)$ satisfy

$$
\left|T C(j)-\frac{R^{n}}{n}\right| \leq \rho-1 \quad \text { for all } 1 \leq j \leq n
$$

In particular, when $n$ is a power of 2 there exist totally-balanced cyclic $n$-digit quaternary Gray codes.

Proof. By hypothesis, $T C(j)-\frac{R^{n}}{n}$ is an integer, and so the strict inequality in the definition of nearly-balanced implies (15).

When $R=4$ and $n=2^{k}, n$ divides $R^{n}$ and so every transition count of a nearlybalanced cyclic $n$-digit quaternary code is within $\rho-1=1$ of the even integer $4^{n} / n$. Since the code is cyclic and $R$ is even, every transition count must be even and so must equal $4^{n} / n$.

We end with an application to binary codes. 
Corollary 2. For each $n \geq 2$ there exists a cyclic $2 n$-digit binary Gray code whose transition counts $T C(1), \ldots T C(2 n)$ are such that every average of the form $\frac{T C(2 i-1)+T C(2 i)}{2}$ equals either $\left\lfloor 2^{2 n} /(2 n)\right\rfloor$ or $\left\lceil 2^{2 n} /(2 n)\right\rceil$.

Proof. Consider the following mapping of elements of $\mathbb{Z}_{4}$ to 2-digit binary strings:

$$
0 \mapsto 00 \quad ; \quad 1 \mapsto 01 \quad ; \quad 2 \mapsto 11 \quad ; \quad 3 \mapsto 10
$$

This mapping extends to a mapping of quaternary $n$-strings to binary $2 n$-strings under which Lee distance is preserved. Therefore, any cyclic $n$-digit quaternary Gray code is mapped to a cyclic $2 n$-digit binary Gray code, and the transition counts $T C(1), \ldots T C(2 n)$ of the binary code have the property that $T C(2 i-1)+T C(2 i)$ equals the transition count of the digit $i$ in the 4 -ary code for every $i=1, \ldots n$. Using any nearly-balanced $n$-digit 4-ary Gray code, we therefore have

$$
\left|\frac{T C(2 i-1)+T C(2 i)}{2}-\frac{2^{2 n}}{2 n}\right|=\frac{1}{2}\left|T C(2 i-1)+T C(2 i)-\frac{4^{n}}{n}\right|<1,
$$

from the definition of nearly-balanced. Since every transition count of a cyclic binary Gray code is even, each such average is an integer and the last inequality implies it must equal either $\left\lfloor 2^{2 n} /(2 n)\right\rfloor$ or $\left\lceil 2^{2 n} /(2 n)\right\rceil$, as claimed.

Acknowledgment: The authors are grateful to the two referees for their helpful suggestions, especially for the simplification of the proof of Theorem 4.

\section{References}

[1] A. Ádám. Truth functions and the problem of their realization by two-terminal graphs. Akadémiai Kiadó, Budapest, 1968. pages 28-37.

[2] Girish S. Bhat and Carla D. Savage. Balanced Gray codes. Electronic Journal of Combinatorics, 3(1):Research Paper 25, approx. 11 pp. (electronic), 1996.

[3] B. Bose and B. Broeg. Lee distance Gray codes. In Proceedings of the International Symposium on Information Theory, 1995.

[4] B. Bose, B. Broeg, Y. Kwon, and Y. Ashir. Lee distance and topological properties of $k$-ary $n$-cubes. IEEE Transactions on Computers, 44:1021-1030, 1995.

[5] Martin Cohn. Affine m-ary Gray codes. Information and Control, 6:70-78, 1963.

[6] Donald E. Knuth. The Art of Computer Programming, volume 4, Fascicle 2. AddisonWesley, 2005.

[7] John P. Robinson and Martin Cohn. Counting sequences. IEEE Transactions on Computers, 30(1):17-23, 1981.

[8] Bhu Dev Sharma and Ravinder Kumar Khanna. On m-ary Gray codes. Inform. Sci., 15(1):31-43, 1978. 
[9] I Nengah Suparta. Counting sequences, Gray codes and Lexicodes. PhD thesis, Delft University of Technology, 2006.

[10] A. J. van Zanten and I. N. Suparta. Totally balanced and exponentially balanced Gray codes. Discrete Analysis and Operations Research, Ser. I, 11:81-98, 2004.

[11] David G. Wagner and Julian West. Construction of uniform Gray codes. Congr. Numer., 80:217-223, 1991. 